\title{
EXTENSIONS OF THE BERGER-SHAW THEOREM
}

\author{
DON HADWIN AND ERIC NORDGREN
}

(Communicated by John B. Conway)

\begin{abstract}
We show how D. Voiculescu's proof of the Berger-Shaw trace inequality for rationally cyclic nearly hyponormal operators can be presented using only elementary operator-theoretic concepts. In addition we show that if $T$ is a hyponormal operator whose essential spectrum has zero area, then the question of whether $\left[T^{*}, T\right]$ is trace class depends only on the spectral picture of $T$. We also show how a special case of results of Helton-Howe can be derived from the BDF theory.
\end{abstract}

In [9] D. Voiculescu proved the following beautiful generalization of the BergerShaw inequality for hyponormal operators.

THEOREM 1. Suppose $T$ is an operator on a Hilbert space such that $\left[T^{*}, T\right]=$ $T^{*} T-T T^{*}$ is the sum of a positive operator and a trace class operator. If $X$ is a Hilbert-Schmidt operator and $T+X$ is rationally $m$-cyclic, then

$$
\pi \operatorname{tr}\left[T^{*}, T\right] \leq m \text { Area } \sigma(T+X) .
$$

The original Berger-Shaw result [3] is the special case when $\left[T^{*}, T\right] \geq 0$ and $X=0$. A remarkable feature of Voiculescu's proof is that it can be presented so that it depends only on elementary operator-theoretic concepts. In this paper we provide such a presentation, and we extend the generalization a bit further. In the case when the essential spectrum $\sigma_{\mathbf{e}}(T)$ of $T$ has area 0 , we show that the spectral picture of $T$ completely determines whether or not $\left[T^{*}, T\right]$ is trace class. In this case we show how some results of Helton and Howe [6] and Pincus [7] can be derived from the Brown-Douglas-Fillmore theory [5]. We also provide a proof of Theorem 1 in the case when $m=\infty$ and Area $\sigma(T+X)=0$; this proof was omitted in [9].

The first step in the proof of Theorem 1 is the following lemma; here $\|\cdot\|_{2}$ denotes the Hilbert-Schmidt norm.

LEMMA 2. If $T$ is an operator and $P$ is a finite-rank projection, then

$$
\operatorname{tr} P\left[T^{*}, T\right] P \leq\|(1-P) T P\|_{2}^{2} .
$$

ProOF. Write $P$ as an operator matrix $P=\left|\begin{array}{ll}1 & 0 \\ 0 & 0\end{array}\right|$ and write $T=\left|\begin{array}{ll}A & B \\ C & D\end{array}\right|$. Then

$$
P\left[T^{*}, T\right] P=\left|\begin{array}{cc}
{\left[A^{*}, A\right]+C^{*} C-B B^{*}} & 0 \\
0 & 0
\end{array}\right| .
$$

Thus

$$
\operatorname{tr} P\left[T^{*}, T\right] P=\operatorname{tr}\left[A^{*}, A\right]+\|C\|_{2}^{2}-\|B\|_{2}^{2} .
$$

Received by the editors April 26, 1985 and, in revised form, November 24, 1986. 1980 Mathematics Subject Classification (1985 Revision). Primary 47B10, 47B20.

The authors gratefully acknowledge support from the NSF during the preparation of this paper. 
Since $A$ is finite dimensional, $\operatorname{tr}\left[A^{*}, A\right]=0$. Hence

$$
\operatorname{tr} P\left[T^{*}, T\right] P \leq\|C\|_{2}^{2}=\|(1-P) T P\|_{2}^{2} .
$$

REMARK. The proof of Lemma 2 shows that

$$
\|(1-P) T P\|_{2}^{2}=\operatorname{tr} P\left[T^{*}, T\right] P+\|P T(1-P)\|_{2}^{2},
$$

and the proof works whenever $\operatorname{tr}\left[A^{*}, A\right]=0$.

LEMMA 3. Suppose $T$ is rationally $m$-cyclic. Then there is a sequence $\left\{P_{k}\right\}$ of finite-rank projections such that $P_{k} \uparrow 1$ and $\operatorname{rank}\left(1-P_{k}\right) T P_{k} \leq m$ for $k=1,2, \ldots$.

ProOF. Let $\left\{f_{1}, f_{2}, \ldots, f_{m}\right\}$ be a ratinally cyclic set for $T$, and let $\left\{z_{1}, z_{2}, \ldots\right\}$ be dense in the complement of $\sigma(T)$. For each positive integer $k$ let $P_{k}$ be the projection onto the subspace spanned by $\left\{T^{j}\left(T-z_{1}\right)^{-1} \cdots\left(T-z_{k}\right)^{-1} f_{i}: 0 \leq j \leq 2 k\right.$ and $1 \leq i \leq m\}$. Since $T$ maps at most $m$ of these vectors out of the range of $P_{k}$, it follows that $\operatorname{rank}\left(1-P_{k}\right) T P_{k} \leq m$. Since arbitrary rational functions of $T$ with poles off $\sigma(T)$ can be approximated arbitrarily closely in norm by finite linear combinations of operators of the form $T^{j}\left(T-z_{1}\right)^{-1} \cdots\left(T-z_{k}\right)^{-1}$, with $0 \leq j \leq 2 k$, it follows that $P_{k} \uparrow 1$.

LEMMA 4. Suppose $\left[T^{*}, T\right]$ is the sum of a positive operator and a trace class operator, and suppose $X$ is a Hilbert-Schmidt operator such that $T+X$ is rationally $m$-cyclic. Then $\operatorname{tr}\left[T^{*}, T\right] \leq m\|T+X\|^{2}$.

ProOF. First apply Lemma 3 to $T+X$ to obtain a sequence $\left\{P_{k}\right\}$ of finite-rank projections such that $P_{k} \uparrow 1$ and such that $\operatorname{rank}\left(1-P_{k}\right)(T+X) P_{k} \leq m$ for $k \geq 1$. Note that it follows that

$$
\left\|\left(1-P_{k}\right)(T+X) P_{k}\right\|_{2}^{2} \leq m\left\|\left(1-P_{k}\right)(T+X) P_{k}\right\|^{2}
$$

for $k \geq 1$. Furthermore, since $X$ is Hilbert-Schmidt, it follows that $\left\|\left(1-P_{k}\right) X P_{k}\right\|_{2}$ $\rightarrow 0$ as $k \rightarrow \infty$. Thus, by Lemma 2 , we have

$$
\begin{aligned}
& \operatorname{tr}\left[T^{*}, T\right]=\lim _{k} \operatorname{tr} P_{k}\left[T^{*}, T\right] P_{k} \leq \limsup _{k}\left\|\left(1-P_{k}\right) T P_{k}\right\|_{2}^{2} \\
& =\limsup _{k}\left\|\left(1-P_{k}\right)(T+X) P_{k}\right\|_{2}^{2} \\
& \leq \limsup _{k} m\left\|\left(1-P_{k}\right)(T+X) P_{k}\right\|^{2} \leq m\|T+X\|^{2} .
\end{aligned}
$$

PROOF OF THEOREM 1 . We need to consider two cases.

Case 1. $m<\infty$. Let $R=\|T+X\|$, and let $D$ be the disk centered at 0 with radius $R$. Suppose $\varepsilon>0$. We can choose a finite disjoint collection $\left\{D_{1}, D_{2}, \ldots, D_{n}\right\}$ of closed disks contained in $D \backslash \sigma(T+X)$ so that Area $(D)<\operatorname{Area} \sigma(T+X)+$ $\sum_{k} \operatorname{Area}\left(D_{k}\right)+\varepsilon$. In other words, if each $D_{k}$ has center $a_{k}$ and radius $r_{k}$, then $\pi R^{2}-\pi \sum_{k} r_{k}^{2}<$ Area $\sigma(T+X)+\varepsilon$. Let $S$ be the unilateral shift operator, and for $1 \leq k \leq n$, let $S_{k}$ be the direct sum of $m$ copies of $a_{k}+r_{k} S$. Then each $S_{k}$ is $m$ cyclic, and since the spectra of the operators $T+X, S_{1}, \ldots, S_{n}$ are pairwise disjoint, it follows that $W=(T+X) \oplus S_{1} \oplus \cdots \oplus S_{n}$ is rationally $m$-cyclic. Clearly $\|W\|=R$. Also, if $V=T \oplus S_{1} \oplus \cdots \oplus S_{n}$, then $\left[V^{*}, V\right]$ is the sum of a positive operator and a trace class operator. Thus, by Lemma 4 , we obtain $\operatorname{tr}\left[V^{*}, V\right] \leq m\|W\|^{2}=m R^{2}$. However,

$$
\operatorname{tr}\left[V^{*}, V\right]=\operatorname{tr}\left[T^{*}, T\right]+\sum_{k} \operatorname{tr}\left[S_{k}^{*}, S_{k}\right]=\operatorname{tr}\left[T^{*}, T\right]+m \sum_{k} r_{k}^{2}
$$


Thus

$$
\pi \operatorname{tr}\left[T^{*}, T\right] \leq m\left(\pi R^{2}-\sum_{k} \pi r_{k}^{2}\right)<m(\text { Area } \sigma(T+X)+\varepsilon) .
$$

Since $\varepsilon$ was arbitrary, the proof is complete in this case.

Case 2. $m=\infty$ and Area $\sigma(T+X)=0$. Clearly, there is no harm in assuming the underlying Hilbert space is separable with an orthonormal basis $\left\{e_{1}, e_{2}, \ldots\right\}$. For each positive integer $n$, let $P_{n}$ be the projection onto the rationally $m$-cyclic invariant subspace for $T+X$ generated by $\left\{e_{1}, \ldots, e_{n}\right\}$. Fix $n$ and write $P_{n}=$ $\left|\begin{array}{ll}1 & 0 \\ 0 & 0\end{array}\right|, T=\left|\begin{array}{ll}A & B \\ C & D\end{array}\right|$ and $X=\left|\begin{array}{cc}V & W \\ Y & Z\end{array}\right|$. Since the range of $P_{n}$ is invariant for $T+X$, we conclude $C=-Y$. Since

$$
P_{n}\left[T^{*}, T\right] P_{n}=\left|\begin{array}{cc}
{\left[A^{*}, A\right]+C^{*} C-B B^{*}} & 0 \\
0 & 0
\end{array}\right|
$$

and $\left[T^{*}, T\right]$ is the sum of a positive operator and a trace class operator, it follows that $\left[A^{*}, A\right]+C^{*} C-B B^{*}$ is the sum of a positive operator and a trace class operator. Since $C=-Y$ is Hilbert-Schmidt, $C^{*} C$ must be a trace class operator. Furthermore since the range of $P_{n}$ is rationally invariant for $T+X$, it follows that $\sigma(A+V) \subset \sigma(T+X)$. Hence $A+V$ is rationally $m$-cyclic and Area $\sigma(A+V)=0$. Applying Case 1 to $A$ and $V$, we see that $\left[A^{*}, A\right]$ is trace class and $\operatorname{tr}\left[A^{*}, A\right] \leq 0$. Thus, as in the proof of Lemma 2, we see

$$
\operatorname{tr} P_{n}\left[T^{*}, T\right] P_{n} \leq\|C\|_{2}^{2}=\left\|\left(1-P_{n}\right) X P_{n}\right\|_{2}^{2} .
$$

Since $P_{n} \uparrow 1$, we obtain $\operatorname{tr}\left[T^{*}, T\right] \leq 0$ by taking the limit as $n \rightarrow \infty$.

REMARK. All of the above proofs are due to D. Voiculescu, except the proof of Lemma 3, in which we modified Voiculescu's argument to avoid using his $q_{2}$ function, and the proof of Case 2 above.

In [4] Berger and Shaw proved a version of Theorem 1 in which rational cyclicity was replaced by effectual rational cyclicity. For vectors $f_{1}, f_{2}, \ldots, f_{m}$ let $\mathfrak{M}\left(T ; f_{1}, f_{2}, \ldots, f_{m}\right)$ be the closure of the set $\left\{f: \exists\right.$ polynomials $q, p_{1}, \ldots, p_{m}$ with $q \neq 0$ such that $\left.q(T) f=p_{1}(T) f_{1}+p_{2}(T) f_{2}+\cdots+p_{m}(T) f_{m}\right\}$. It is easily seen that $\mathfrak{M}\left(T ; f_{1}, f_{2}, \ldots, f_{m}\right)$ is a subspace that is invariant under $r(T)$ for every rational function $r$ with poles off $\sigma(T)$. An operator $T$ is effectually rationally $m$-cyclic if there are vectors $f_{1}, f_{2}, \ldots, f_{m}$ such that $\mathfrak{M}\left(T ; f_{1}, f_{2}, \ldots, f_{m}\right)$ is the whole Hilbert space. This definition agrees with the one in [4] but differs slightly from the one in [2] in which a version of Theorem 1 for von Neumann algebras is established.

We now turn to a generalization of Theorem 1 that is inspired by Lemmas 3 and 4. For each operator $T$ let $\tau(T)$ be the lim inf of $\operatorname{rank}(1-P) T P$ as $P$ ranges over the finite-rank projections and $P \rightarrow 1$ in the strong operator topology. The lim inf here is obtained by taking the infimum of all $\operatorname{rank}((1-P) T P)$ as $P$ ranges over all finite-rank projections in a strong operator topology neighborhood of 1 and then taking the supremum of such infima over all neighborhoods of 1 . Since the relative strong operator topology on the set of projections is metrizable, the lim inf may also be characterized as the smallest of the sequential limits, $\lim _{n} \operatorname{rank}\left(\left(1-P_{n}\right) T P_{n}\right)$, as $\left(P_{n}\right)$ varies over all sequences of finite-rank projections converging strongly to 1 for which the limit exists. Suppose $\tau(T)<\infty$. Since rank is cardinal-valued, there is a sequence of finite-rank projections $P_{n}$ such that $P_{n} \rightarrow 1$ and $\operatorname{rank}\left(1-P_{n}\right) T P_{n}=$ $\tau(T)$ for $n=1,2, \ldots$ The content of Lemma 3 is that if $T$ is rationally $m$-cyclic, 
then $\tau(T) \leq m$. Furthermore, the proof of Lemma 4 shows that if $\left[T^{*}, T\right]$ is the sum of a positive operator and a trace class operator, and if $X$ is a Hilbert-Schmidt operator, then $\operatorname{tr}\left[T^{*}, T\right] \leq \tau(T+X)\|T+X\|^{2}$. Our aim is to prove the following analog of Theorem 1.

THEOREM 5. If $\left[T^{*}, T\right]$ is the sum of a positive operator and a trace class operator, and if $X$ is a Hilbert-Schmidt operator, then

$$
\pi \operatorname{tr}\left[T^{*}, T\right] \leq \tau(T+X) \text { Area } \sigma(T+X) .
$$

An examination of the proof of Theorem 1 (Case 1) shows that to prove Theorem 5 we need only show that if $A$ and $B$ are operators with disjoint spectra, then $\tau(A \oplus B) \leq \max (\tau(A), \tau(B))$. Therefore Theorem 5 is a consequence of the following proposition, which contains some of the basic properties of the function $\tau$. Part (1) of the following shows why it would be natural to call $\tau(T)$ the modulus of triangularity of $T$ (see [1] and [10]).

PROPOSITION 6. Suppose $S, T$ and $Y$ are operators and $m$ is a positive integer. Then

(1) $\tau(T)=0$ if and only if $T$ is upper triangular with respect to some orthonormal basis;

(2) $\tau(S \oplus T) \leq \tau(S)+\tau(T)$;

(3) if $\left\{Q_{n}\right\}$ is a sequence of projections onto invariant subspaces of $T$ such that $Q_{n} \rightarrow 1$ then $\tau(T) \leq \liminf _{n} \tau\left(T \mid \operatorname{ran}\left(Q_{n}\right)\right)$;

(4) if $T$ is effectually rationally $m$-cyclic, then $\tau(T) \leq m$;

(5) $\tau(T) \leq m$ if and only if there is a sequence $\left\{Q_{n}\right\}$ of projections with $Q_{n} \rightarrow 1$ in the strong operator topology such that the range of each $Q_{n}$ is the sum of a finite-dimensional invariant subspace of $T$ and an $m$-cyclic invariant subspace of $T$;

(6) $\tau(T) \leq m$ if and only if there is a sequence $\left\{Q_{n}\right\}$ of finite-rank projections with $Q_{n} \rightarrow 1$ in the strong operator topology such that the range of each $Q_{n}$ generates an invariant subspace for $T$ on which $T$ is effectually rationally $m$-cyclic;

(7) if $\sigma(S) \cap \sigma(T)=\varnothing$, then $\tau(S \oplus T)=\max (\tau(S), \tau(T))$;

(8) if $S Y=Y T$ and $Y$ has dense range, then $\tau(S) \leq \tau(T)$; and

(9) if $S$ and $T$ are quasisimilar, then $\tau(S)=\tau(T)$.

PROOF. Statements (1), (2) and (3) follow from the definition of $\tau$.

(4) Suppose $T$ is effectually rationally $m$-cyclic. Then there are vectors $f_{1}, f_{2}, \ldots$, $f_{m}$ such that $\left\{g\right.$ : for some $n, T^{n+1} g$ is in the linear span of $\left\{T^{k} g: 0 \leq k \leq\right.$ $n\} \cup\left\{T^{k} f_{j}: k \geq 0\right.$ and $\left.\left.1 \leq j \leq m\right\}\right\}=\mathfrak{M}$ is dense. Let $\left\{g_{1}, g_{2}, \ldots\right\}$ be dense in $\mathfrak{M}$. For each positive integer $s$ there are positive integers $n(s)$ and $k(s)$ such that, for $1 \leq i \leq s$, the vector $T^{n(s)+1} g_{i}$ is in the linear span of $\left\{T^{k} g_{i}: 0 \leq k \leq\right.$ $n(s)\} \cup\left\{T^{k} f_{j}: 0 \leq k \leq k(s)\right.$ and $\left.1 \leq j \leq m\right\}$. Let $P_{s}$ be the projection onto the linear span of $\left\{T^{k} g_{i}: 0 \leq k \leq n(s)\right.$ and $\left.1 \leq i \leq s\right\} \cup\left\{T^{k} f_{j}: 0 \leq k \leq k(s)\right.$ and $1 \leq j \leq m\}$. As in the proof of Lemma 3, we see that $\operatorname{rank}\left(1-P_{s}\right) T P_{s} \leq m$ for $s \geq 1$. Clearly $P_{s} \rightarrow 1$ as $s \rightarrow \infty$. Hence $\tau(T) \leq m$.

(5) The "if" part of (5) is a consequence of (3) and (4). Conversely, suppose that $P$ is a finite-rank projection and $\operatorname{rank}(1-P) T P=m \geq 1$. Let $\mathfrak{M}$ be the range of $P$. We shall show that the smallest invariant subspace of $T$ that incudes $\mathfrak{M}$ is the 
sum of a finite-dimensional invariant subspace and an $m$-cyclic invariant subspace. This will imply the "only if" part of (5).

First note that $m$ is the dimension of the quotient space $T(\mathfrak{M}) / \mathfrak{M} \cap T(\mathfrak{M})$. For each $f$ in $\mathfrak{M}$, define the order of $f$ to be the smallest positive integer $n$ for which $T^{n} f$ is not in $\mathfrak{M}$, provided such an integer exists; otherwise define the order of $f$ to be $\infty$. If $f$ is in $\mathfrak{M}$, then either the order of $f$ is infinite or else it is no greater than $\operatorname{dim} \mathfrak{M}$, and $\mathfrak{M}_{0}=\{f \in \mathfrak{M}: f$ has infinite order $\}$ is a finite-dimensional invariant subspace of $T$. Let $f_{1}$ be a vector in $\mathfrak{M}$ having maximal finite order $n(1)$. Let $f_{2}$ be a vector in $\mathfrak{M}$ having maximal finite order $n(2)$ among all the vectors $f$ of finite order $n$ such that $T^{n} f$ and $T^{n(1)} f_{1}$ are linearly independent modulo $\mathfrak{M}$. Proceeding inductively, we can choose from $\mathfrak{M}$ vectors $f_{1}, f_{2}, \ldots, f_{m}$ (since $\operatorname{dim} T(\mathfrak{M}) / \mathfrak{M} \cap T(\mathfrak{M})=m$ ) so that, for each $k, 1 \leq k \leq m$, the vector $f_{k}$ has maximal order among all vectors $f$ of finite order $n$ such that $T^{n} f, T^{n(1)} f_{1}, \ldots, T^{n(k-1)} f_{k-1}$ are linearly independent modulo $\mathfrak{M}$. Let $\mathfrak{N}$ be the sum of $\mathfrak{M}_{0}$ and the linear span of $\left\{T^{k} f_{i}: 0 \leq k<n(i)\right.$ and $1 \leq i \leq m\}$. We will show that $\mathfrak{N}=\mathfrak{M}$. If $\mathfrak{N} \neq \mathfrak{M}$, then there is a vector $f$ in $\mathfrak{M}$ such that $f$ has maximal (finite) order $n$ among those vectors in $\mathfrak{M}$ but not in $\mathfrak{N}$. Let $k$ be the smallest positive integer for which $T^{n} f, T^{n(1)} f_{1}, \ldots, T^{n(k)} f_{k}$ are linearly dependent modulo $\mathfrak{M}$. It is clear from the choice of $f_{i}$ 's that $n \leq n_{k} \leq$ $\cdots \leq n_{1}$. There are scalars $a_{1}, a_{2}, \ldots, a_{k}$ such that $T^{n} f-\sum_{i} a_{i} T^{n(i)} f_{i}$ is in $\mathfrak{M}$. Let $h=f-\sum_{i} a_{i} T^{n(i)-n} f_{i}$. Then $T^{n} h \in \mathfrak{M}$, and $T^{j} h \in \mathfrak{M}$ for $0 \leq j<n$. Thus the order of $h$ is greater than $n$. It follows from the choice of $n$ that $h \in \mathfrak{N}$; whence $f=h-\sum_{i} a_{i} T^{n(i)-n} f_{i} \in \mathfrak{N}$. This contradiction implies that $\mathfrak{M}=\mathfrak{N}$. It follows that the smallest closed invariant subspace of $T$ that includes $\mathfrak{M}$ is the sum of $\mathfrak{M}_{0}$ and the $m$-cyclic invariant subspace generated by $\left\{f_{1}, f_{2}, \ldots, f_{m}\right\}$. This completes the proof of (5).

(6) The "if" part of (6) is implied by (3) and (4) and the "only if" part by (5).

(7) If $\sigma(S) \cap \sigma(T)=\varnothing$, then by Runge's theorem $1 \oplus 0$ is in the norm closed algebra generated by the rational functions in $S \oplus T$. Thus, by the remarks preceding the definition of effectual rational $m$-cyclic, for vectors $f_{1}, f_{2}, \ldots, f_{m}$ and $g_{1}, g_{2}, \ldots, g_{m}$ it follows that

$\mathfrak{M}\left(S \oplus T ; f_{1} \oplus g_{1}, f_{2} \oplus g_{2}, \ldots, f_{m} \oplus g_{m}\right)=\mathfrak{M}\left(S ; f_{1}, f_{2}, \ldots, f_{m}\right) \oplus \mathfrak{M}\left(T ; g_{1}, g_{2}, \ldots, g_{m}\right)$.

Hence (7) is implied by (6).

(8) Suppose $S Y=Y T$ and $Y$ has dense range. Since $p(S) Y=Y p(T)$ for every polynomial $p$, it follows that if $\mathfrak{M}$ is a finite-dimensional invariant subspace for $T$, then $Y(\mathfrak{M})$ is invariant for $S$, and if $\mathfrak{M}$ is an $m$-cyclic invariant subspace for $T$, then the closure of $Y(\mathfrak{M})$ is an $m$-cyclic invariant subspace for $S$. It follows from (5) that if $\tau(T) \leq m$, then there is a sequence $\left\{Q_{n}\right\}$ of projections such that $Q_{n} \rightarrow 1$ in the strong operator topology and the range of each $Q_{n}$ is the sum of a finite-dimensional invariant subspace of $T$ and an $m$-cyclic invariant subspace of $T$. Thus if $P_{n}$ is the projection onto the closure of the range of $Y Q_{n}$, then the range of each $P_{n}$ is the sum of a finite-dimensional invariant subspace of $S$ and an $m$-cyclic invariant subspace for $S$. Since $Y$ has dense range, it follows that $P_{n} \rightarrow 1$ strongly, and (5) implies $\tau(S) \leq m$.

(9) Clearly, (8) implies (9).

REMARK. In the case when $T$ is normal, it follows from a beautiful, deep result of $\mathrm{D}$. Voiculescu that there is a Hilbert-Schmidt operator $X$ such that $T+X$ is 
diagonal [11]. Since $\tau(T+X)=0$, we see that Theorem 5 gives the best possible estimate for $\operatorname{tr}\left[T^{*}, T\right]$, while Theorem 1 does not.

We now turn to a generalization of Theorem 1 that does not depend on any sort of cyclicity, but rather on the spectral picture of the operator. Suppose $T$ is an operator with essential spectrum $\sigma_{\mathrm{e}}(T)$. Let $V_{1}, V_{2}, \ldots$ be the bounded components of the complement of $\sigma_{\mathrm{e}}(T)$, and let $m_{1}, m_{2}, \ldots$ be the associated Fredholm indices (i.e., $m_{k}=\operatorname{dim} \operatorname{ker}(T-z)-\operatorname{dim} \operatorname{ker}(T-z)^{*}$ for every $z$ in $\left.V_{k}, k=1,2, \ldots\right)$. The Weyl essential spectrum $\sigma_{\mathrm{we}}(T)$ of $T$ is the union of $\sigma_{\mathrm{e}}(T)$ with those $V_{k}$ 's for which $m_{k} \neq 0$. It was proved by Stampfli [8] that there is a compact operator $K$ such that $\sigma(T+K)=\sigma_{\mathrm{we}}(T)$. Moreover in the first step of Stampfli's proof he shows that there there is a Hilbert-Schmidt operator $X$ such that $\sigma(T+X) \backslash \sigma_{\text {we }}(T)$ is countable. Using Stampfli's result and Theorem 1 we obtain the following result.

COROLLARY 7. If $\left[T^{*}, T\right]$ is the sum of a positive operator and a trace operator, and if Area $\sigma_{\mathrm{we}}(T)=0$, then $\left[T^{*}, T\right]$ is trace class and $\operatorname{tr}\left[T^{*}, T\right]=0$.

PROOF. By the above remarks, choose a Hilbert-Schmidt operator $X$ such that $\sigma(T+X) \backslash \sigma_{\text {we }}(T)$ is countable. Thus Area $\sigma(T+X)=0$. It follows from Theorem 1 that $\operatorname{tr}\left[T^{*}, T\right] \leq 0$; thus $\left[T^{*}, T\right]$ is trace class. Hence we may apply our argument to $T^{*}$ to obtain $\operatorname{tr}\left[T^{*}, T\right] \geq 0$.

THEOREM 8. Suppose $\left[T^{*}, T\right]$ is the sum of a positive operator and a trace class operator. Let $V_{1}, V_{2}, \ldots$ be the bounded components of the complement of $\sigma_{\mathrm{e}}(T)$, with associated Fredholm indices $m_{1}, m_{2}, \ldots$ Suppose also that

(1) Area $\sigma_{\mathrm{e}}(T)=0$, and

(2) $\sum\left\{\left|m_{k}\right|\right.$ Area $\left.V_{k}: m_{k}<0\right\}<\infty$.

Then

(3) $\left[T^{*}, T\right]$ is trace class,

(4) $\sum_{k}\left|m_{k}\right|$ Area $V_{k}<\infty$, and

(5) $\pi \operatorname{tr}\left[T^{*}, T\right]=-\sum_{k} m_{k}$ Area $V_{k}$.

PROOF. For each $k$ with $m_{k} \neq 0$, choose a seqence $\left\{D_{k n}\right\}$ of open disks with disjoint closures contained in $V_{k}$ so that $\operatorname{Area}\left(V_{k} \backslash \bigcup_{n} D_{k n}\right)=0$. For each such $k$ and each $n$, suppose $D_{k n}$ has center $a_{k n}$ and radius $r_{k n}$, and let $S_{k}$ be a direct sum of $\left|m_{k}\right|$ copies each of the operators $S_{k n}$ defined by

$$
S_{k n}= \begin{cases}a_{k n}+r_{k n} S & \text { if } m_{k}>0, \\ a_{k n}+r_{k n} S^{*} & \text { if } m_{k}<0,\end{cases}
$$

where $S$ is the unilateral shift. Let $W=S_{1} \oplus S_{2} \oplus \cdots$. Then $\left[W^{*}, W\right]$ is a diagonal operator, and the sum of the absolute values of the negative eigenvalues of $\left[W^{*}, W\right]$ is

$$
\begin{aligned}
\sum\left\{\sum_{n}\left|m_{k}\right| r_{k n}^{2}: m_{k}<0\right\} & =\frac{1}{\pi} \sum\left\{\left|m_{k}\right| \sum_{n} \text { Area } D_{k n}: m_{k}<0\right\} \\
& =\frac{1}{\pi} \sum\left\{\left|m_{k}\right| \text { Area } V_{k}: m_{k}<0\right\}
\end{aligned}
$$

which is finite by (2) above. Thus $\left[(T \oplus W)^{*},(T \oplus W)\right]$ is the sum of a positive operator and a trace class operator. Furthermore, $\sigma_{\mathrm{e}}(T \oplus W)=\sigma_{\mathrm{e}}(T) \cup \sigma_{\mathrm{e}}(W)$, and $\sigma_{\mathrm{e}}(W)$ is contained in the union of $\sigma_{\mathrm{e}}(T)$ with the sets $V_{k} \backslash \bigcup_{n} D_{k n}\left(m_{k} \neq 0\right)$. Hence, by (1) and the choice of the $D_{k n}$ 's, we see that Area $\sigma_{\mathrm{e}}(T \oplus W)=0$. Furthermore, 
the Fredholm index of $(T \oplus W)-z$ is 0 for each $z$ in one of the $D_{k n}$ 's and for each $z$ in one of the $V_{k}$ 's with $m_{k}=0$. Hence $\sigma_{\text {we }}(T \oplus W)=\sigma_{\mathrm{e}}(T \oplus W)$, which has area 0 . Thus, by Corollary $7,\left[(T \oplus W)^{*},(T \oplus W)\right]$ is trace class and has trace 0 . Thus $\left[T^{*}, T\right]$ and $\left[W^{*}, W\right]$ are trace class operators and

$$
\begin{aligned}
\pi \operatorname{tr}\left[T^{*}, T\right] & =-\pi \operatorname{tr}\left[W^{*}, W\right]=-\pi \sum_{k, n}\left|m_{k}\right| \operatorname{sgn}\left(m_{k}\right) r_{k n}^{2} \\
& =-\sum_{k} m_{k} \sum_{k} \text { Area } D_{k n}=-\sum_{k} m_{k} \text { Area } V_{k} .
\end{aligned}
$$

This proves (3) and (5). Clearly (4) follows from (2), (3) and (5).

REMARK. It was shown by Helton and Howe [6] and by Pincus [7] that, among the statements in the above theorem, (3) implies (4) (and hence (2)), and that (1) and (3) together imply (5). Thus half of the following corollary depends on [6 and 7].

COROLlaRY 9. Suppose that $\left[T^{*}, T\right]$ is the sum of a positive operator and a trace class and that Area $\sigma_{\mathrm{e}}(T)=0$. Then $\left[T^{*}, T\right]$ is trace class if and only if $\sum\left\{\left|m_{k}\right|\right.$ Area $\left.V_{k}: m_{k}<0\right\}<\infty$.

COROLlARY 10. Suppose that $\left[T^{*}, T\right]$ is the sum of a positive operator and a trace class operator and that Area $\sigma_{\mathrm{e}}(T)=0$. Then $\pi \operatorname{tr}\left[T^{*}, T\right] \leq \sum\left\{\left|m_{k}\right|\right.$ Area $V_{k}$ : $\left.m_{k}<0\right\}$.

COROLLRY 11. Suppose that $\left[T^{*}, T\right]$ is the sum of a positive operator and a trace class operator and that Area $\sigma_{\mathrm{e}}(T)=0$. Then

$$
\pi \operatorname{tr}\left[T^{*}, T\right] \leq\left(\sup _{k}-m_{k}\right) \text { Area } \sigma_{\mathrm{we}}(T) .
$$

REMARK. It follows from part (4) of Proposition 6 that $\tau(T) \geq \sup _{k}-m_{k}$ for every operator $T$. Hence, in the case when Area $\sigma_{\mathrm{e}}(T)=0$, Corollary 11 is an improvement of Theorem 5 (and Theorem 1).

COROLLARY 12. Suppose that $\left[T^{*}, T\right]$ is the sum of a positive operator and a trace class operator and that Area $\sigma_{\mathrm{e}}(T)=0$. If the complement of $\sigma_{\mathrm{e}}(T)$ has only finite many components, then $\left[T^{*}, T\right]$ is trace class.

COROLlaRY 13. If $\left[T^{*}, T\right]$ and $\left[S^{*}, S\right]$ are each the sum of a positive operator and a trace class operator and that Area $\sigma_{\mathrm{e}}(T)=0$, and if there is an essentially unitary operator $U$ such that $U^{*} T U-S$ is compact, then $\left[T^{*}, T\right]$ is trace class if and only if $\left[S^{*}, S\right]$ is trace class and $\operatorname{tr}\left[T^{*}, T\right]=\operatorname{tr}\left[S^{*}, S\right]$.

We turn now to a result of Helton and Howe [6] and Pincus [7]. Suppose $\left[T^{*}, T\right]$ is trace class and $T=X+i Y$ with $X$ and $Y$ Hermitian. In [6] Helton and Howe constructed a finite real Borel measure $P$ on $\sigma(T)$ such that if $p(x, y)$ and $q(x, y)$ are polynomials, then

$$
2 \pi i \operatorname{tr}[p(X, Y), q(X, Y)]=\int_{\sigma(T)} J(p, q) d P
$$

where $J(p, q)=(\partial p / \partial x)(\partial q / \partial y)-(\partial p / \partial y)(\partial q / \partial x)$ is the Jacobian of $p$ and $q$. Since $2 i[X, Y]=\left[T^{*}, T\right]$ is trace class, $X$ and $Y$ commute modulo the trace class 
operators. Thus evaluations of $p(X, Y)$ or $q(X, Y)$ in more than one way (i.e., letting the variables $x$ and $y$ commute) differ by only a trace class operator, which does not affect the trace in (\#).

Pincus [7] proved that the measure $P$ is absolutely continuous with respect to area measure, and it was shown in [6] that the Radon-Nikodým derivative $d P / d$ Area takes the value $-m_{k}$ on each component $V_{k}$ of the complement of $\sigma_{\mathrm{e}}(T)$. In the case when Area $\sigma_{\mathbf{e}}(T)=0$, it follows that (\#) becomes

(\#\#)

$$
2 \pi i \operatorname{tr}[p(X, Y), q(X, Y)]=-\sum_{k} m_{k} \int_{V_{k}} J(p, q) d x d y
$$

Note that letting $p(x, y)=x$ and $q(x, y)=y$ in (\#\#) yields Theorem 8(5).

We conclude by showing how Theorem 8 , combined with [5], yields a short proof of (\#\#) under the additional assumption that $\sum_{k}\left|m_{k}\right|$ Area $V_{k}<\infty$. This latter assumption is redundant, by Corollary 9, but the proof of the relevant half of Corollary 9 uses the results in [6]. It would be nice to find an elementary proof that $\sum_{k}\left|m_{k}\right|$ Area $V_{k}<\infty$ whenever $\left[T^{*}, T\right]$ is trace class.

THEOREM 14. Suppose $\left[T^{*}, T\right]$ is trace class and let $V_{1}, V_{2}, \ldots$ be the components of the complement of $\sigma_{\mathrm{e}}(T)$ with associated Fredholm indices $m_{1}, m_{2}, \ldots$ Assume that Area $\sigma_{\mathrm{e}}(T)=0$ and $\sum_{k}\left|m_{k}\right|$ Area $V_{k}<\infty$, and write $T=X+i Y$ with $X$ and $Y$ Hermitian. For each pair $p(x, y)$ and $q(x, y)$ of polynomials, we have

$$
2 \pi i \operatorname{tr}[p(X, Y), q(X, Y)]=-\sum_{k} m_{k} \int_{V_{k}} J(p, q) d x d y
$$

PROOF. In [6, pp. 150-153], an elementary proof of the theorem is given in the case when $T=a+r S$ or $T=a+r S^{*}$, where $S$ is the unilateral shift and $r>0$. For any operator $T$, let $\phi(T)$ denote the left side of (\#\#).

It follows from polarization that we can assume that $p(X, Y)=q(X, Y)^{*}$. Let the $D_{k n}$ 's the $S_{k n}$ 's and $W$ be as in the proof of Theorem 8, and let $A=T \oplus W$. Then $\left[A^{*}, A\right]$ is trace class, Area $\sigma_{\mathrm{e}}(A)=0$, and the Fredholm index of $A-z$ is zero for every $z$ in the complement of $\sigma_{\mathrm{e}}(A)$. It follows from [5] that $A$ is the sum of a normal operator and a compact operator. If $A=B+i C$ with $B$ and $C$ Hermitian, then $q(B, C)$ is the sum of a normal operator and a compact operator. Thus the Fredholm index of $q(B, C)-z$ is zero for every $z$ not in $\sigma_{\mathbf{e}}(q(B, C))=\left\{q(x, y): x+i y \in \sigma_{\mathbf{e}}(A)\right\}$, which has area 0 . Thus, by Theorem $8, \phi(A)=0\left(\right.$ since $\left.p(B, C)=q(B, C)^{*}\right)$.

Hence

$$
\begin{aligned}
\phi(T) & =-\phi(W)=-\sum_{k}\left|m_{k}\right| \sum_{n} \phi\left(S_{k n}\right) \\
& =-\sum_{k} \sum_{n}\left|m_{k}\right| \operatorname{sgn}\left(m_{k}\right) \int_{D_{k n}} J(p, q) d x d y \\
& =-\sum_{k} m_{k} \int_{V_{k}} J(p, q) d x d y .
\end{aligned}
$$

Question. Is $d P / d$ Area $\leq \tau(T)$ a.e.? 


\section{REFERENCES}

1. C. Apostol, Quasitriangularity in Hilbert space, Indiana Univ. Math. J. 22 (1973), 817-825.

2. C. A. Berger and M. G. Ben-Jacob, Trace-class self-commutators, Trans. Amer. Math. Soc. 277 (1983), 75-91.

3. C. A. Berger and B. I. Shaw, Self-commutators of multicyclic hyponormal operators are always trace class, Bull. Amer. Math. Soc. 79 (1973), 1193-1199.

4.

5. L. Brown, R. G. Douglas and P. A. Fillmore, Unitary equivalence modulo the compact operators and extensions of $C^{*}$-algebras, Lecture Notes in Math., vol. 345, Springer-Verlag, Berlin and New York, 1973, pp. 58-128.

6. J. W. Helton and R. E. Howe, Integral operators: commutators, traces, index, and homology, Lecture Notes in Math., vol. 345, Springer-Verlag, Berlin and New York, 1973, pp. 141-209.

7. J. D. Pincus, On the trace of commutators in the algebra generated by an operator with trace class self-commutator, Stony Brook Preprint, 1972.

8. J. G. Stampfli, Compact perturbations, normal eigenvalues and a problem of Salinas, J. London Math. Soc. (2) 9 (1974-1975), 165-175.

9. D. Voiculescu, $A$ note on quasitrianguliarity and trace-class self commutators, Acta Sci. Math. (Szeged) 42 (1980), 195-199.

10 D. Voiculescu, Some extensions of quasitriangularity, Rev. Roumaine Math. Pures Appl. 18 (1973), 1303-1320.

11. _ Some results on norm-ideal perturbations of Hilbert space operators, J. Operator Theory 2 (1979), 3-37.

Department of Mathematics, University of New Hampshire, DuRham, NeW HAMPSHIRE 03824 